\title{
A Regenerative Approach for Root Canal Treatment of Mature Permanent Teeth: Comparative Evaluation with 18 Months Follow-up
}

\author{
Preeti Jha ${ }^{1}$, Mandeep S Virdi ${ }^{2}$, Suman Nain ${ }^{3}$
}

\begin{abstract}
Aim: SealBio is a novel technique which stimulates the periradicular cells to deposit a biological barrier at the root apex by inducing healing and regeneration. This clinical trial was undertaken to compare the outcome of teeth treated with the SealBio and the obturation technique. Materials and methods: Thirty patients met the inclusion criteria and consented to participate in the study. Patients were randomly assigned to the SealBio and the obturation group. The time taken for both the techniques and periapical healing was evaluated. The patients of both the groups were evaluated at 6,12, and 18 months follow up. The periapical index (PAI) was the primary outcome measure to check the apical bone density and healing. The secondary outcome measure was the presence/absence of signs and symptoms. The final outcome measure was the sum of the primary and secondary outcome measures.

Results and conclusion: The time taken to perform endodontic treatment with the SealBio technique was significantly lesser than that of obturation. Both groups showed equally favorable outcomes at the end of 18 months without any statistically significant differences.

Clinical significance: The results of the present study have demonstrated that SealBio technique gives similar results as that of conventional gutta-percha obturation. The shortcomings of obturation such as difficulty in obtaining a fluid-tight seal and difficulty in obturating tortuous canals can be overcome by the SealBio method. The SealBio method is cost effective, less technique sensitive, and takes lesser chair time.

Keywords: Apical periodontitis, Mature teeth, Necrotic pulp, Periapical abscess, Regenerative endodontics, SealBio.

International Journal of Clinical Pediatric Dentistry (2019): 10.5005/jp-journals-10005-1616
\end{abstract}

\section{INTRODUCTION}

Effective sealing at both the apical and coronal ends of a disinfected root canal system are the essential requisites of a successful root canal treatment. ${ }^{1}$ Gutta-percha along with a sealer has been the material of choice for obturating the root canals. ${ }^{2}$ The periapex contains a variety of progenitor cells which may participate in the regeneration of tissues as a response to injury. ${ }^{3}$ Thus, regeneration rather than replacement of tissues with an artificial substitute is the alternative direction in which endodontics is progressing.

The idea of revascularization is not new, perse. It was conceptualized as early as 1961, by Östby ${ }^{4}$ and, in 1966, root development and apical barrier formation subsequent to pulpal necrosis in children were demonstrated by Rule and Winter. ${ }^{5}$ Complete maturation of the immature root of necrotic teeth following the revascularization protocol as compared to the formation of a calcific barrier in conventional calcium hydroxide apexification has been demonstrated. ${ }^{6}$

An empty canal space itself will not aid in the growth of tissues from the periapex. ${ }^{7}$ Revascularization studies have used blood or blood substitutes, collagen solutions, bovine collagen with calcium, and phosphate crystals as scaffolds that have been able to aid in the regeneration of tissues. ${ }^{8}$ However, the formation of granulation tissue upon inducing bleeding into the canals forms a more favorable environment with vascular cells which allows for self-renewal of stem cells and progenitors, thus, providing a niche for the future dental progeny. ${ }^{3}$

The SealBio technique is a novel, regeneration-based protocol. It is based on the concept of revascularization and depends on the regenerative potential of the stem cells present in the periradicular area of the root apex. On stimulation, these cells lead to the deposition of a biological barrier at the root apex following healing
${ }^{1-3}$ Department of Pedodontics and Preventive Dentistry, PDM Dental College and Research Institute, Bahadurgarh, Haryana, India

Corresponding Author: Mandeep S Virdi, Department of Pedodontics and Preventive Dentistry, PDM Dental College and Research Institute, Bahadurgarh, Haryana, India, Phone: +91 9810704958, e-mail: mandeepsingh74@gmail.com

How to cite this article: Jha P, Virdi MS, et al. A Regenerative Approach for Root Canal Treatment of Mature Permanent Teeth: Comparative Evaluation with 18 Months Follow-up. Int J Clin Pediatr Dent 2019;12(3):182-188.

Source of support: Nil

Conflict of interest: None

and regeneration. ${ }^{2}$ This approach tries to achieve a biological seal rather than a mechanical seal, unlike artificial obturating materials. This in vivo study was undertaken to compare the outcome of the teeth treated with the SealBio and obturation technique.

\section{Materials and Methods}

\section{Subject Enrolment}

The present randomized controlled clinical trial was approved by the Ethical Committee and the institutional review board. Study subjects were recruited from the pool of patients referred to the Department of Pedodontics and Preventive Dentistry for initial nonsurgical root canal treatment. Thirty cases of periapical infection with completely formed roots with a radiographic evidence of periapical pathology in subjects between 9 and 15 years of age irrespective of gender or the tooth involved were included in the study. Teeth that could be 
isolated under rubber dam, which showed radiographic evidence of apical periodontitis (Ostravik's PAI score $\geq 3$ ) and gave a negative response to cold and electric pulp tests, were included in the study. Patients with systemic disease, incompletely formed roots, having a history of antibiotic use within the past month, or with known allergy to any of the constituents of the triple antibiotic paste were excluded from the study. After the written informed consent was obtained, the patients were randomly assigned to either the SealBio (group I) or the obturation (group II) group.

\section{Clinical Procedures}

All the procedures for both the groups were performed by the same operator. After achieving local anesthesia and rubber dam isolation, a standard access cavity was prepared. For both groups, initial canal working length was established by using the Propex II electronic apex locator (Dentsply) and a \#15 stainless steel K file which was then confirmed radiographically. In group I, conventional biomechanical preparation was done using the Dentsply rotary protaper universal files along with negative pressure irrigation (Endovac, Discus dental), using $2.5 \%$ sodium hypochlorite $(\mathrm{NaOCl})$. The apical third of the canals was debrided by "apical clearing" followed by "apical foramen widening" with larger K-files used sequentially to size $25-30$ as mentioned in the SealBio technique patented by Shah et al. ${ }^{2}$ Subsequent to biomechanical preparation of the canals in group I, the triple antibiotic paste ${ }^{9}$ was placed in the canals for 1 or 2 weeks until the teeth became asymptomatic.

When the infection control was achieved, as evident from the absence of signs and symptoms, the SealBio technique was performed. The teeth were anesthetized by using 3\% mepivacaine without adrenaline and were isolated under a rubber dam. The antibiotic dressing was removed from the canals by agitating the dressing with hand files and irrigating with $17 \%$ ethylenediaminetetraacetic acid (EDTA). After checking the patency, determined by a smooth passage of \#15 ISO instrument, intentional over-instrumentation into the periapical region was done with the sterile \#20 K-file to induce bleeding near the apical foramen. The file was gently given two to three clock-wise turns and then withdrawn by giving counter clockwise rotation. Calcium sulfate-based cement (Cavit G) was introduced in the access cavity with a hand plugger and was condensed into the cervical third of root canals. A suitable coronal restoration was given and an immediate posttreatment radiograph was made.

In group II, conventional biomechanical preparation was done using the Dentsply rotary protaper universal files and negative pressure irrigation with $2.5 \% \mathrm{NaOCl}$. Once the tooth became asymptomatic, it was obturated by the conventional cold lateral condensation technique.

\section{Recording the Time}

The timing for the procedures was noted after the teeth were anesthetized and isolated using a rubber dam till the completion of the SealBio procedure before placing the coronal restoration in group I and till the completion of the obturation procedure before placing the coronal restoration in group II using a stopwatch. The patients of both the groups were recalled at 6,12 , and 18 months interval for clinical and radiographic evaluation.

\section{Evaluation of Treatment Outcome}

The evaluation of treatment outcome for this study was done with the help of the primary outcome measure, i.e., $\mathrm{PAI}^{10}$ and secondary outcome measure (clinical signs and symptoms) at 6, 12, and 18 months follow-up. The final outcome was determined by combining the primary and secondary outcome measures. ${ }^{11}$

\section{Primary Outcome Measure}

The primary outcome measure for this study was change in apical bone density at subsequent follow-ups. The PAI was used to evaluate radiographic healing..$^{10}$ All the pre- and postoperative radiographs were made using the bite registration as a positioning index ${ }^{12}$ keeping the same exposure settings on the same imaging system each time. The radiographic examination was performed by three blinded independent examiners using coded radiographs in which the root canals of the teeth were masked. ${ }^{12}$ Before the consensus meeting, the intra- and the interexaminer the variability were found to $0.83-0.89$ and 0.91 , respectively, which suggested almost perfect agreement.

\section{Secondary Outcome Measure}

Secondary outcome measures were the presence or absence of clinical signs and symptoms at 6, 12, and 18 months (spontaneous pain, presence of sinus tract, swelling, mobility, periodontal probing depths greater than baseline measurements, or sensitivity to percussion or palpation) which were recorded for both the groups.

\section{Final Outcome Measure}

The final treatment outcome was interpreted at the 18th month follow-up by combining the primary and the secondary outcome measure using the criteria: ${ }^{11}$

Healed-combined radiographic and clinical normalcy

Healing - reducing radiolucency with clinical normalcy

Diseased-if the radiolucency persisted without change with or without clinical normalcy.

\section{Statistical Analysis}

The time taken for the endodontic treatment, intra- and interrater agreement, PAI scores, and the final treatment outcome were subjected to statistical analysis. Data were analyzed using SPSS version 16. The time taken to complete the endodontic treatment by the SealBio and obturation technique was statistically analyzed using the Student $t$ test. The agreement within and between the examiners was determined by using the intraclass correlation coefficient. The PAl scores for both the groups at preoperative, 6,12 , and 18 months follow-up and the final treatment outcome at 18 months were statistically analyzed by using Pearson's Chi-square test.

\section{Results}

Table 1 shows the mean time taken for endodontic treatment. In group I, the mean time taken was $16.02 \mathrm{~min}$ and, in group II, it was $36.59 \mathrm{~min}$. The differences in time taken for both the groups were statistically significant $(p<0.05)$. Table 2 represents the mean PAI score of groups I and II preoperatively and at 6, 12, and 18 months follow-up. In group I, the mean preoperative PAI score was 3.6 which decreased to 1.6 at 6 months, 1.4 at 12 months, and 1.1 at 18 months follow-up. In group II, the mean preoperative PAI score was 3.4 which decreased to 1.73 at 6 months, 1.4 at 12 months, and 1.2 at 17 months follow-up (graphical representation in Fig. 1). The difference between the mean PAI score for both the groups at each interval was not statistically significant $(p<0.05)$. Table 3 represents the final treatment outcome at the end of 18 months. Thirteen out of 15 teeth completely healed in group I (SealBio), whereas 12 out 
Table 1: Mean time taken for the endodontic treatment in groups I and II

\begin{tabular}{llcll}
\hline Group & Mean $(\mathrm{min})$ & Std. deviation & t value & $p$-value \\
\hline SealBio & 16.02 & 7.60 & 4.992 & $<0.001$ \\
Obturation & 36.59 & 14.39 & & \\
\hline
\end{tabular}

Table 2: Mean PAI score of groups I and II preoperatively and at 6, 12, and 18 months follow-up

\begin{tabular}{|c|c|c|c|c|}
\hline & SealBio $(I)$ & Obturation (II) & $\begin{array}{l}\text { Pearson } \\
\text { Chi-square }\end{array}$ & $p$-value \\
\hline Preoperative & $3.6 \pm 0.97$ & $3.4 \pm 0.82$ & 0.682 & 0.409 \\
\hline At 6 months & $1.6 \pm 1.05$ & $1.73 \pm 1.09$ & 1.053 & 0.789 \\
\hline At 12 months & $1.4 \pm 0.73$ & $1.4 \pm 0.82$ & 2.243 & 0.326 \\
\hline At 18 months & $1.1 \pm 0.35$ & $1.2 \pm 0.41$ & 0.24 & 0.62 \\
\hline
\end{tabular}

Table 3: Final treatment outcome at 18 months for groups I and II

\begin{tabular}{|c|c|c|c|c|c|}
\hline & Healed & Healing & Diseased & $\begin{array}{l}\text { Pearson } \\
\text { Chi-square }\end{array}$ & $p$-value \\
\hline SealBio (I) & $13 / 15$ & $2 / 15$ & $0 / 15$ & 0.24 & 0.62 \\
\hline Obturation (II) & $12 / 15$ & $3 / 15$ & $0 / 15$ & & \\
\hline
\end{tabular}

of 15 teeth completely "healed" in group II (obturation), 2 out of 15 teeth in group I and 3 in group II were considered as "healing." None of the groups showed any evidence of persisting disease. There was no statistically significant difference in the final treatment outcome between the two groups $(p<0.05)$ (Fig. 2).

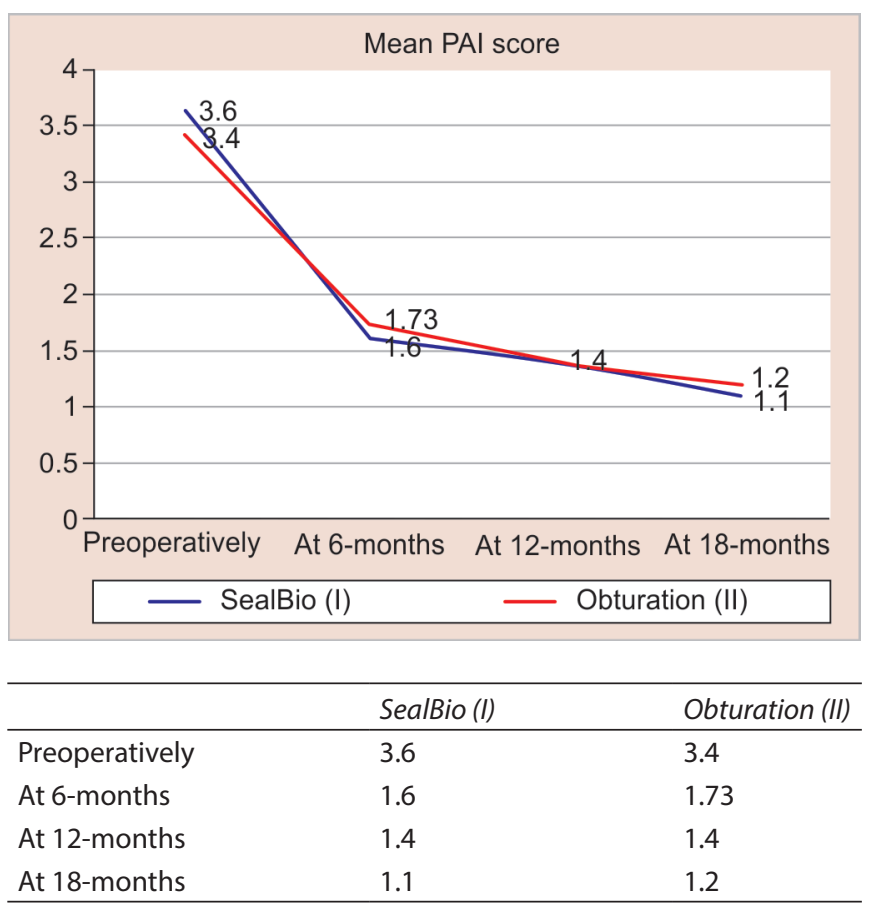

Fig. 1: Graphical presentation of the mean PAI scores of groups I and II preoperatively and at 6,12 , and 18 months follow-up. Actual numbers from which a graph is drawn
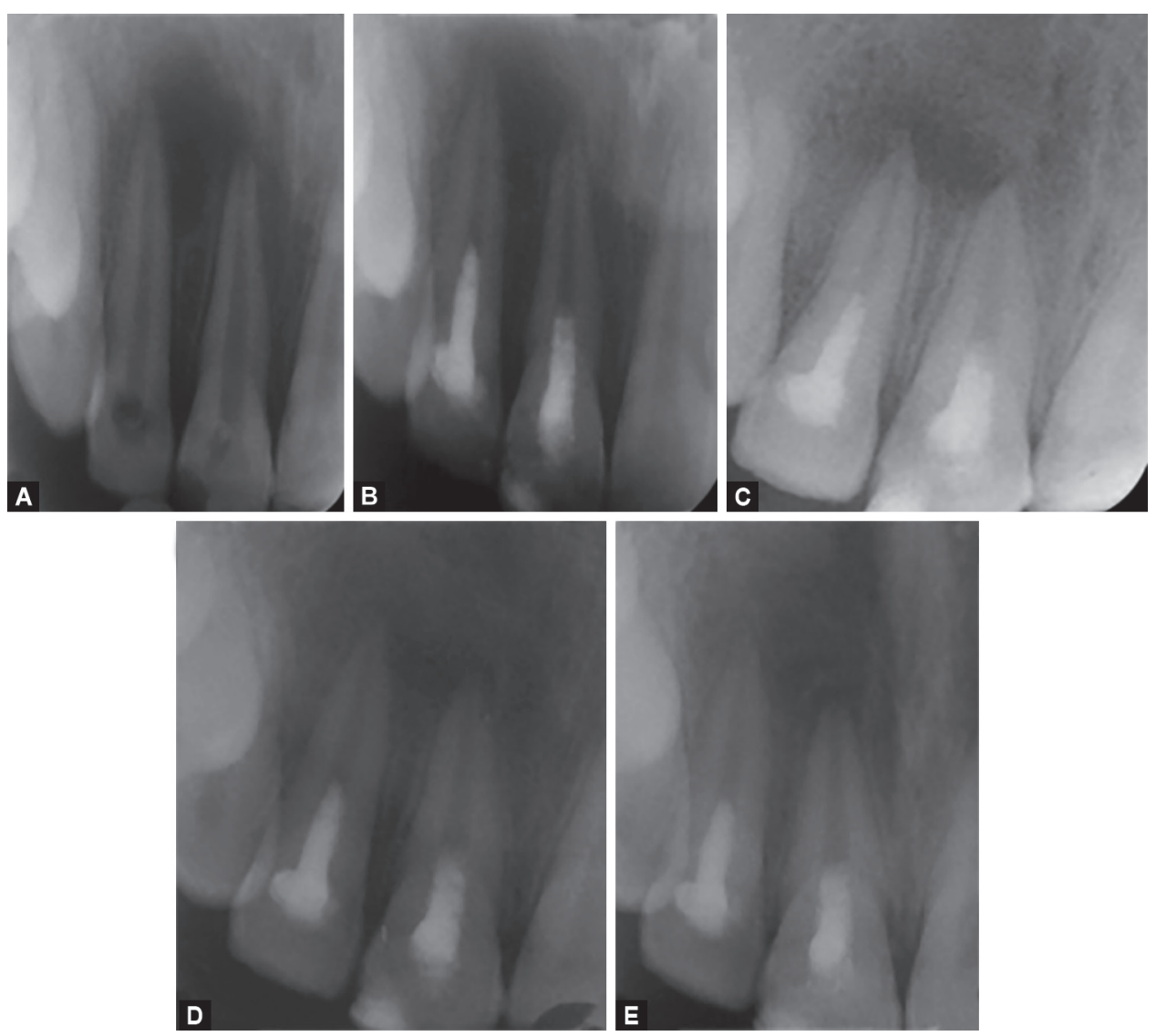

Figs $2 \mathrm{~A}$ to E: (A) Preoperative radiograph of \#7 and \#8 showing large periapical radiolucent lesion; (B) Immediate postoperative radiograph after the SealBio procedure; (C) 6-months follow-up; (D) 12-months follow-up; (E) 18-months follow-up 


\section{Discussion}

The outcome of a root canal treatment is believed to be based on the sterilization of the canal system and achieving a hermetic seal. ${ }^{13}$ However, some doubt exists about whether it is possible to achieve a hermetic seal with the available root canal filling materials. ${ }^{14}$ Dubrow ${ }^{15}$ suggested that failure following root canal filling could be attributed to an inadequate technique during the entire treatment and that reaming, cleaning, and disinfecting the canal alone could produce a favorable response. Theoretically, if we can sterilize the canal and prevent coronal leakage, then the entire root canal filling may not be necessary. ${ }^{16}$ Shah and Logani patented the regeneration-based SealBio technique in $2009,{ }^{2}$ and it has been tested over the past 6 years on 134 teeth in 116 patients. ${ }^{17}$ In spite of numerous successful case reports, ${ }^{1,17-21}$ no comparative study in humans has been published. Our results are in accordance with a comparative study between obturated and nonobturated canals in dogs carried out by Sabeti et al. which did not show any significant difference in healing between the two groups. ${ }^{22}$

In our study, 30 patients were randomly divided into two groups. An attempt was made to equalize the number of multirooted teeth in each group to reduce the bias in the time taken to perform the endodontic treatment. ${ }^{23}$ The time was recorded after the teeth were anesthetized. In the SealBio group, anesthesia was achieved using 3\% mepivacaine. It has been demonstrated that using a local anesthetic with a vasoconstrictor reduces the pulpal blood flow. ${ }^{24}$ Three percent mepivacaine without vasoconstrictor has shown to facilitate the flow of blood into the canals following intentional overinstrumentation in the periapical area of the tooth and is similar in efficacy when compared with $2 \%$ lidocaine with 1:100,000 epinephrine. ${ }^{25,26}$ The results of our study showed that a statistically significant shorter time was needed for the SealBio technique. The mean time for the SealBio technique was $16.02 \pm 7.60$ minutes and the mean time for obturation was $36.59 \pm 14.39$ minutes. The shorter time can be attributed to the elimination of steps like, choosing a gutta percha with "tug back" and taking a master cone X-ray (Fig. 3).

The preoperative PAI score of three or greater than three was taken as primary inclusion criteria for this study. The initial PAI scores for both the groups were approximately equal in distribution with a mean of 3.6 in the SealBio group and 3.4 in obturation group which was not statistically significant. The number of single rooted and multirooted was equalized during randomization because single-rooted teeth with apical periodontitis have a higher probability of complete healing when compared with multirooted teeth which could have led to the source of bias. ${ }^{27}$ The procedures were performed by a single operator for both the groups. The operator was a second-year pedodontics resident.
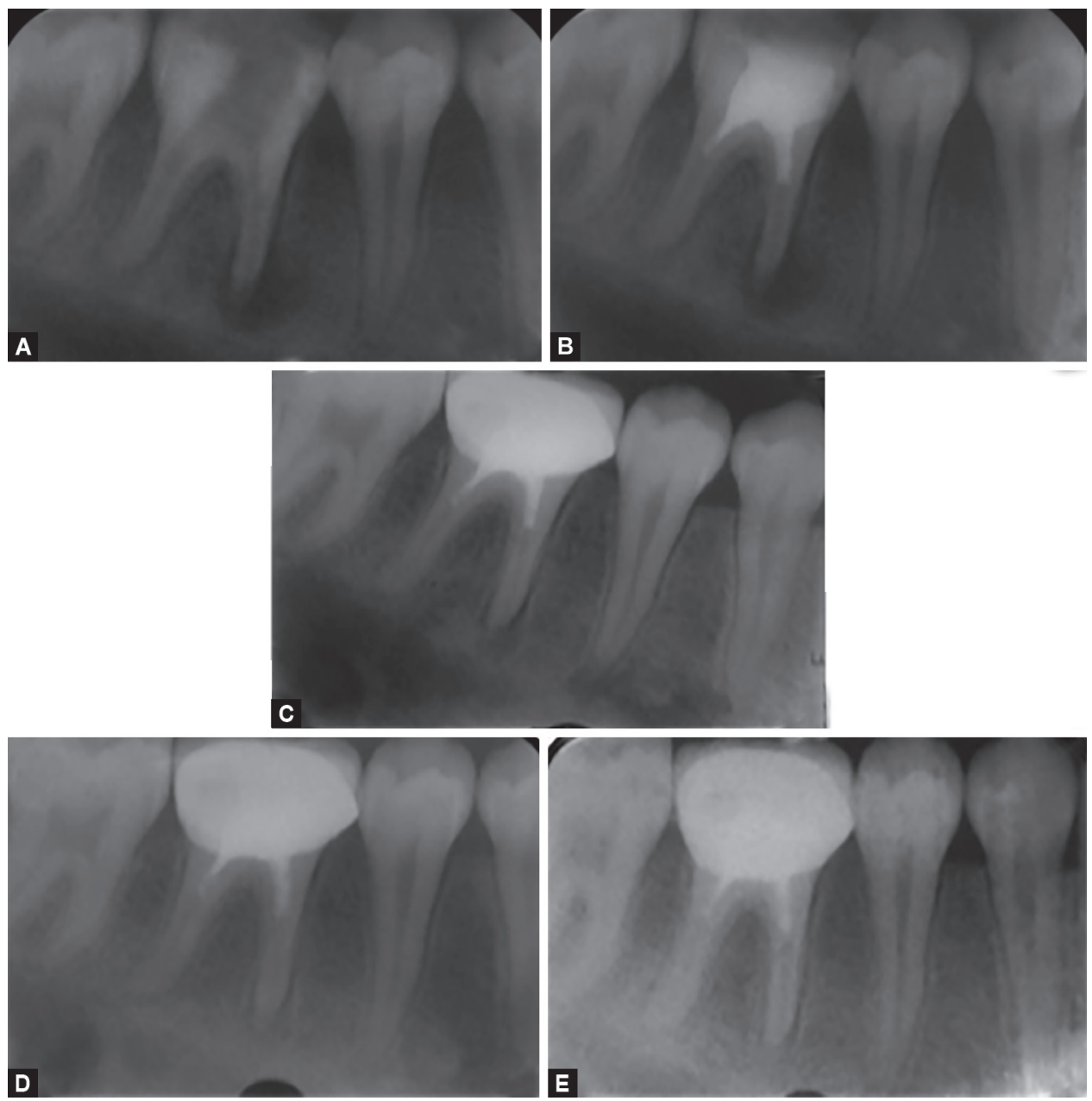

Figs 3A to E: (A) Preoperative radiograph of \#30 showing well-defined periapical radiolucent lesion at the mesial root; (B) Immediate postoperative radiograph after the SealBio procedure; (C) 6-months follow-up; (D) 12-months follow-up; (E) 18-months follow-up 
All the procedures were performed under faculty supervision, consultation, and assistance.

The patients included in the study were followed up at 6-month intervals for 18 months. The periapical healing is a dynamic process and sufficient time is required to evaluate its progression and completion. However, a reversal of the healing process is a rare phenomenon. ${ }^{27}$ Healing of apical periodontitis peaks within the first year; however, complete resolution of the periapical radiolucency may take 4-5 years and follow-up of at least 1 year is required to reveal meaningful changes. ${ }^{27}$ Teeth that demonstrate signs of reducing radiolucency for up to two consecutive followups can forecast complete healing at a later time. ${ }^{28}$ Although longer observation periods might be ideal, if periapical changes in bone density by using PAl is evident at 18 months then longer follow-up might not be necessary. The PAI score reduced from 3.6 preoperatively to 1.1 in the SealBio group and 3.4-1.2 in the obturation group. The interrater reliability score of 0.91 shows very a high level of agreement between examiners which supports the reliability of PAI in measuring apical bone density radiographically (Fig. 4).

To determine a strict definition of success, both radiographic (PAI) and clinical findings were combined to assess the final outcome of the treatment which was categorized into healed, healing, and diseased category at the 18-month follow-up. ${ }^{11}$ In the SealBio group, 13 out of 15 teeth had completely healed at the end of 18 months which was $86.6 \%$ of the original sample and $14.4 \%$ of the sample was healing. In the obturation group, 12 out of 15 teeth showed complete healing which was $80 \%$ of the total sample and $20 \%$ of the sample was healing. The major finding of the present study was that there was no statistically significant difference in the healing of periapical pathosis between the two groups.

One of the main reasons for success in both the groups was the maintenance of a sterilized canal system with the help of a coronal seal. The importance of a tight coronal seal cannot be overemphasized. In both groups, all teeth were sealed by a glass ionomer base followed by a composite restoration. In case of extensive loss of tooth structure, full coverage crowns, either stainless steel or porcelain-fused metal crowns, were given, whereas in anterior teeth when a minimal amount of coronal tooth structure was lost, a direct resin composite restoration was done. ${ }^{29}$ The other reason for success can be attributed to the use of negative pressure irrigation system. Negative pressure irrigation system was used for copiously irrigating the canals with $2.5 \%$ sodium hypochlorite in both the groups. A medium concentration, i.e., $2.5 \% \mathrm{NaOCl}$ was used as a tradeoff between its toxic effects and antimicrobial activity. ${ }^{30}$ Negative pressure irrigation reduced the risk of apical extrusion of the irrigant and provided a better microbial control (Fig. 5). ${ }^{31}$

The success of the unconventional SealBio technique can also be attributed to the placement of triple antibiotic paste in the canals which reduces the bacterial load tremendously by penetrating the dentinal tubules. ${ }^{32}$ Apical clearing and apical foramen widening further help to facilitate healing by removing infected cementum
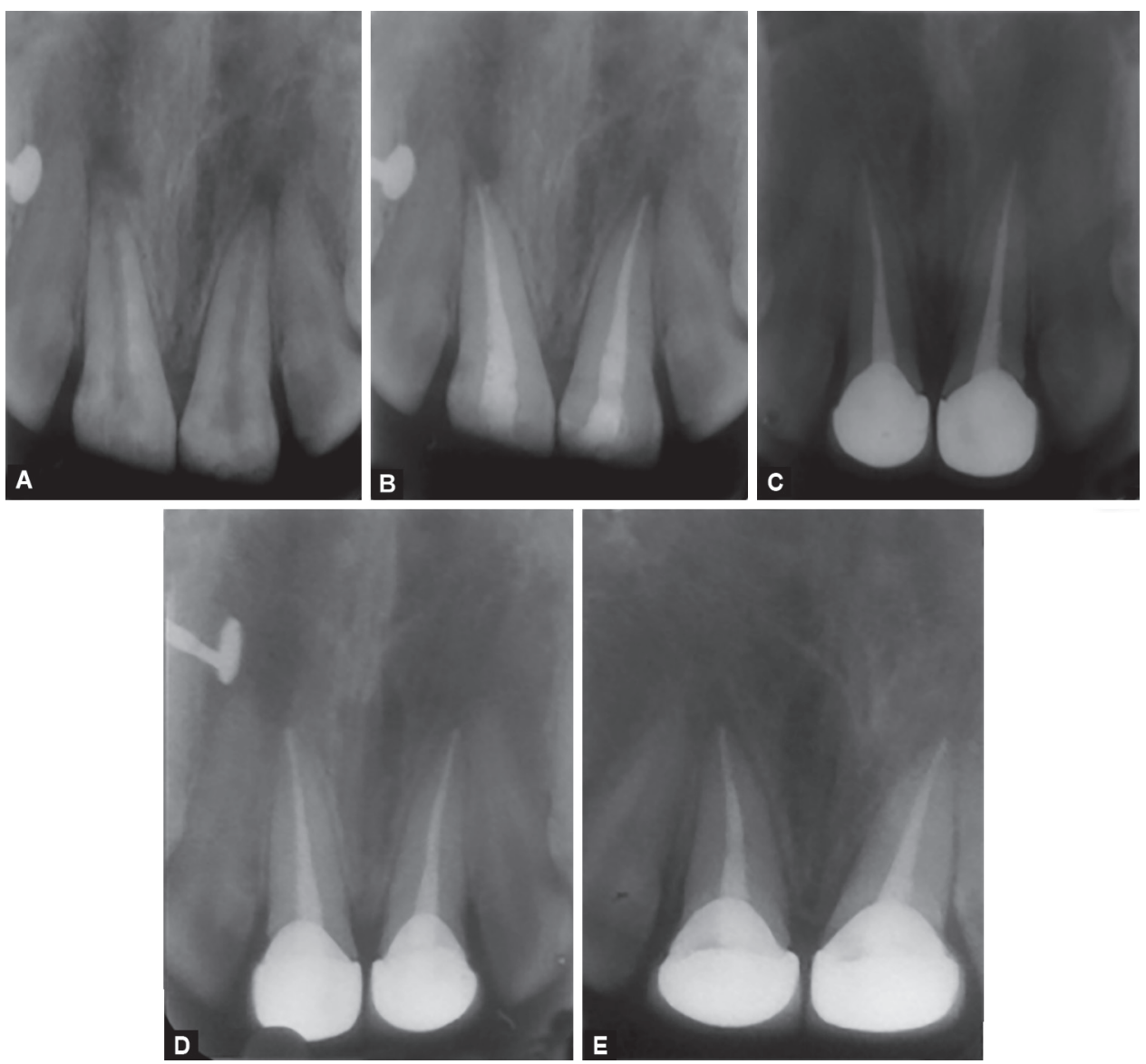

Figs 4A to E: (A) Preoperative radiograph of \#8 and \#9 showing periapical radiolucent lesion; (B) Immediate postoperative radiograph after obturation; (C) 6-months follow-up; (D) 12-months follow-up; (E) 18-months follow-up 

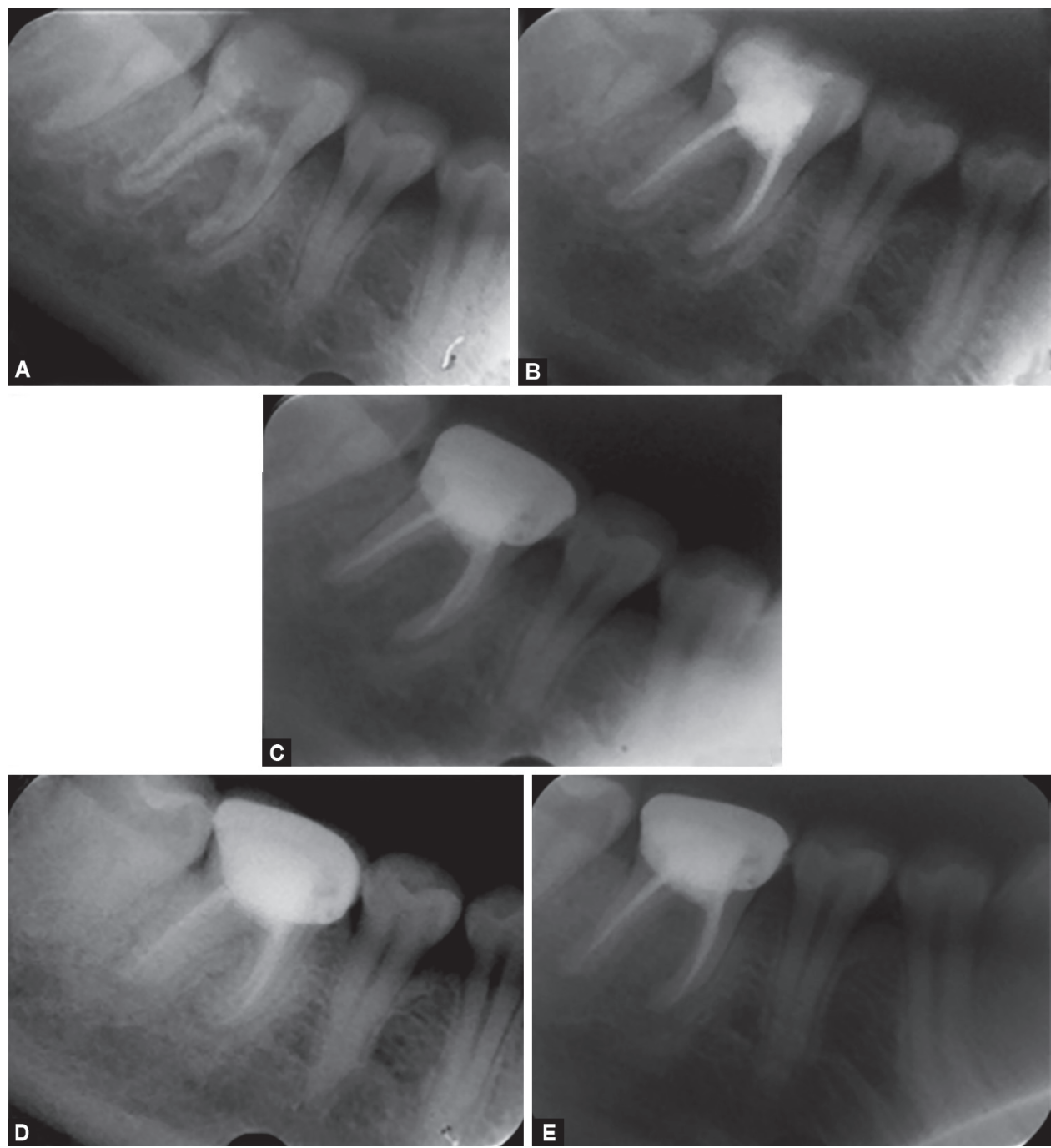

Figs 5A to E: (A) Preoperative radiograph of \#30 showing well defined periapical radiolucent lesion; (B) Immediate postoperative radiograph after the obturation procedure; (C) 6-months follow-up; (D) 12-months follow-up; (E) 18-months follow-up

and any newly deposited cementum or newly grown connective tissue that may have sealed off the apical foramen or accessory canals inside the apical root canal. ${ }^{33}$ It has been hypothesized that widening the apical foramen to 300-500 $\mu$ thoroughly cleanses the apical portion of the canal where bacteria might have penetrated to a depth of $150-250 \mu$ and remain protected from the action of medicament and irrigants. ${ }^{1,33}$ It also helps in the removal of microorganisms from the apical deltas, ramifications, dentinal tubules, and cementum, thus, promoting more favorable conditions for healing. ${ }^{33}$

The bleeding and clot formed in the area of apical foramen by over-instrumentation can lead to seeding of stem cells such as the periodontal ligament stem cells (SCPDL), bone marrow mesenchymal stem cells (BMMSC), and stem cells from apical papilla (SCAP), their proliferation, differentiation, and mineralized tissue formation, thus, sealing the apical foramen. ${ }^{1}$ Also included are components of the innate and adaptive immune system, such as antibacterial molecules (complement components and immunoglobulins), phagocytes (polymorphonuclear leukocytes and macrophages), antimicrobial peptides, and cytokines. ${ }^{34}$ The induction of periapical bleeding enhances antimicrobial clearance in the canals by immune defense mechanisms of generated vital tissue which is supported by the high success rate of regenerative endodontic procedures in immature teeth with necrotic pulp and apical periodontitis. ${ }^{35}$

The results of the present study have demonstrated that SealBio gives similar results as that of conventional gutta-percha obturation. The shortcomings of obturation such as difficulty in obtaining a fluid-tight seal, difficulty in obturating tortuous canals can be overcome by the SealBio method. It increases the emphasis on the coronal seal, cleaning, and disinfection of canals, and proves that the teeth with periapical radiolucencies will heal even in the absence of an obturating material. This method is cost effective, less technique sensitive, and takes lesser chair time. However, the SealBio technique is a novel method and investigations regarding the kind of hard tissue that deposits at the apex need to be carried out using cone beam computed tomography $(\mathrm{CBCT})$ and histological studies. The sample size in the present study was small; the SealBio technique needs to be assessed in larger sample sizes with longer follow-ups before we can include this promising novel regenerative technique in our daily treatment protocol. Nevertheless, it is a promising regenerative alternative to the conventional obturation technique. 


\section{Conclusion}

The results of our trial conclude that the time taken to complete the endodontic treatment with the SealBio technique was significantly shorter as compared to that of obturation. The signs and symptoms resolved and patients remained asymptomatic throughout the period of follow-up in both the groups. There was no statistically significant difference in radiographic evidence of periapical healing between the two groups. The final outcome of both the groups was similar and did not show any statistically significant difference.

\section{Clinical Significance}

The results of the present study have demonstrated that the SealBio technique gives similar results as that of conventional gutta-percha obturation. The shortcomings of obturation such as difficulty in obtaining a fluid-tight seal and difficulty in obturating tortuous canals can be overcome by the SealBio method. The SealBio method is cost effective, less technique sensitive, and takes lesser chair time.

\section{References}

1. Shah N, Logani A. SealBio: a novel, non-obturation endodontic treatment based on concept of regeneration. J Conserv Dent 2012 Oct;15(4):328-332. DOI: 10.4103/0972-0707.101889.

2. Shah N, Logani A. SealBio: A novel, non-obturation endodontic treatment based on concept of regeneration. United States Patent Application Publication, Naseem et al. Pub. No: US 2012/0231422 A1. Pub. Date: Sep 13, 2012.

3. Andreasen JO, Andreasen FM, et al. Textbook and Color Atlas of Traumatic Injuries to the Teeth. Blackwell Publishing Ltd, Oxford, United Kingdom, 4th edn, 2013.

4. Östby BN. The role of the blood clot in endodontic therapy: An experimental histologic study. Acta Odontol Scand 1961 Jan 1;19(3-4):323-353. DOI: 10.3109/00016356109043395.

5. Rule DC, Winter GB. Root growth and apical repair subsequent to pulpal necrosis in children. Br Dent J 1966 Jun 21;120(12):586.

6. Banchs F, Trope M. Revascularization of immature permanent teeth with apical periodontitis: new treatment protocol? J Endod $2004 \mathrm{Apr}$ 30;30(4):196-200. DOI: 10.1097/00004770-200404000-00003.

7. Thibodeau B, Teixeira F, et al. Pulp revascularization of immature dog teeth with apical periodontitis. J Endod 2007 Jun 30;33(6):680. DOI: 10.1016/j.joen.2007.03.001

8. Ding RY, Cheung GS, et al. Pulp revascularization of immature teeth with apical periodontitis: a clinical study. J Endod 2009 May 31;35(5):745-749. DOI: 10.1016/j.joen.2009.02.009.

9. Hoshino E. Information on LSTR 3Mix-MP therapy [Internet]. 2009 [updated 2015 July 7; cited 2017 Feb 28]. 2009, Available from: http:// www.lstr.jp/e/information.html.

10. Ørstavik D, Kerekes K, et al. The periapical index: a scoring system for radiographic assessment of apical periodontitis. Dent Traumatol 1986 Feb 1;2(1):20-34. DOI: 10.1111/j.1600-9657.1986.tb00119.x.

11. Friedman S. Prognosis of initial endodontic therapy. Endod Topics 2002 Jul 1;2(1):59-88. DOI: 10.1034/j.1601-1546.2002.20105.x.

12. Penesis VA, Fitzgerald PI, et al. Outcome of one-visit and two-visit endodontic treatment of necrotic teeth with apical periodontitis: a randomized controlled trial with one-year evaluation. J Endod 2008 Mar 31;34(3):251-257. DOI: 10.1016/j.joen.2007.12.015.

13. Seltzer $S$, Bender IB, et al. Factors affecting successful repair after root canal therapy. J Am Dent Assoc 1963 Nov 30;67(5):651-662. DOI: 10.14219/jada.archive.1963.0349.

14. Younis $\mathrm{O}$, Hembree JH. Leakage of different root canal sealants. Oral Surg Oral Med Oral Pathol 1976 Jun 30;41(6):777-784. DOI: 10.1016/0030-4220(76)90192-4.
15. Dubrow H. Silver points and gutta-percha and the role of root canal fillings. J Am Dent Assoc 1976 Nov 30;93(5):976-980. DOI: 10.14219/ jada.archive.1976.0029.

16. Asgary S, Fazlyab M. A Successful Endodontic Outcome with NonObturated Canals. Iran Endod J 2015;10(3):208.

17. Shah N. A regeneration-based, nonobturation root-canal treatment for fully-mature teeth: six years' experience with "SealBio". Contemp Clin Dent 2016 Jul;7(3):296. DOI: 10.4103/0976-237X.188541.

18. Paryani K, Kim SG. Regenerative endodontic treatment of permanent teeth after completion of root development: a report of 2 cases. J Endod 2013 Jul 31;39(7):929-934. DOI: 10.1016/j.joen.2013.04.029.

19. Saoud TM, Sigurdsson A, et al. Treatment of a large cyst like inflammatory periapical lesion associated with mature necrotic teeth using regenerative endodontic therapy. J Endod 2014 Dec 31;40(12):2081-2086. DOI: 10.1016/j.joen.2014.07.027.

20. Saoud TM, Huang GT, et al. Management of teeth with persistent apical periodontitis after root canal treatment using regenerative endodontic therapy. J Endod 2015 Oct 31;41(10):1743-8. DOI: 10.1016/j.joen.2015.07.004.

21. Saoud TM, Martin G, et al. Treatment of mature permanent teeth with necrotic pulps and apical periodontitis using regenerative endodontic procedures: a case series. J Endod 2016 Jan 31;42(1):57-65. DOI: 10.1016/j.joen.2015.09.015.

22. Sabeti MA, Nekofar $M$, et al. Healing of apical periodontitis after endodontic treatment with and without obturation in dogs. J Endod 2006 Jul 31;32(7):628-633. DOI: 10.1016/j.joen.2005.12.014.

23. Bender IB, Seltzer $S$, et al. To culture or not to culture? Oral Surg Oral Med Oral Pathol 1964 Oct 31;18(4):527-540. DOI: 10.1016/00304220(64)90403-7.

24. Chng HS, Pitt Ford TR, et al. Effects of prilocaine local anaesthetic solutions on pulpal blood flow in maxillary canines. Dent Traumatol 1996 Apr 1;12(2):89-95. DOI: 10.1111/j.1600-9657.1996.tb00103.x.

25. Petrino JA, Boda KK, et al. Challenges in regenerative endodontics: a case series. J Endod 2010 Mar 31;36(3):536-541. DOI: 10.1016/ j.joen.2009.10.006.

26. McLean C, Reader A, et al. An evaluation of $4 \%$ prilocaine and $3 \%$ mepivacaine compared with $2 \%$ lidocaine (1:100,000 epinephrine) for inferior alveolar nerve block. J Endod 1993 Mar 31;19(3):146-150. DOI: 10.1016/S0099-2399(06)80510-8.

27. Ørstavik D. Time course and risk analyses of the development and healing of chronic apical periodontitis in man. Int Endod J 1996 May 1;29(3):150-155. DOI: 10.1111/j.1365-2591.1996.tb01361.x.

28. Friedman S, Löst C, et al. Evaluation of success and failure after endodontic therapy using a glass ionomer cement sealer. J Endod 1995 Jul 31;21(7):384-390. DOI: 10.1016/S0099-2399(06)80976-3.

29. Hargreaves KM, Berman LH. Pathways of Pulp. Elsevier, Philadelphia, 11th edn, 2016.

30. Vianna $\mathrm{ME}, \mathrm{Horz} \mathrm{HP}$, et al. In vivo evaluation of microbial reduction after chemomechanical preparation of human root canals containing necrotic pulp tissue. Int Endod J 2006 Jun 1;39(6):484-492. DOI: 10.1111/j.1365-2591.2006.01121.x.

31. Hockett JL, Dommisch JK, et al. Antimicrobial efficacy of two irrigation techniques in tapered and nontapered canal preparations: an in vitro study. J Endod 2008 Nov 30;34(11):1374-1377. DOI: 10.1016/ j.joen.2008.07.022.

32. Takushige T, Cruz EV, et al. Endodontic treatment of primary teeth using a combination of antibacterial drugs. Int Endod J 2004 Feb 1;37(2):132-138. DOI: 10.1111/j.0143-2885.2004.00771.x.

33. Borlina SC, de Souza V, et al. Influence of apical foramen widening and sealer on the healing of chronic periapical lesions induced in dogs' teeth. Oral Surg Oral Med Oral Pathol Oral Radiol Endod 2010 Jun 30;109(6):932-940. DOI: 10.1016/j.tripleo.2010.01.028.

34. Abbas AK, Lichtman AH, et al. Cellular and Molecular Immunology. Saunders, Philadelphia, 6th edn, 2010.

35. Diogenes A, Henry MA, et al. An update on clinical regenerative endodontics. Endod Topics 2013 Mar 1;28(1):2-3.DOI: 10.1111/etp.12040. 